\title{
Worry is associated with robust reductions in heart rate variability: a transdiagnostic study of anxiety psychopathology
}

\author{
John A. Chalmers ${ }^{1}$, James A. J. Heathers ${ }^{1,2}$, Maree J. Abbott ${ }^{1}$, Andrew H. Kemp ${ }^{1,3,4}$ and Daniel S. Quintana ${ }^{1,5,6^{*}}$
}

\begin{abstract}
Background: Individuals with anxiety disorders display reduced resting-state heart rate variability (HRV), although findings have been contradictory and the role of specific symptoms has been less clear. It is possible that HRV reductions may transcend diagnostic categories, consistent with dimensional-trait models of psychopathology. Here we investigated whether anxiety disorders or symptoms of anxiety, stress, worry and depression are more strongly associated with resting-state HRV.

Methods: Resting-state HRV was calculated in participants with clinical anxiety $(n=25)$ and healthy controls $(n=58)$. Symptom severity measures of worry, anxiety, stress, and depression were also collected from participants, regardless of diagnosis.

Results: Participants who fulfilled DSM-IV criteria for an anxiety disorder displayed diminished HRV, a difference at trend level significance ( $p=.1$, Hedges' $g=-.37, \mathrm{BF}_{10}=.84$ ). High worriers (Total $n=41 ; n=22$ diagnosed with an anxiety disorder and $n=19$ not meeting criteria for any psychopathology) displayed a robust reduction in resting state HRV relative to low worriers ( $p=.001$, Hedges' $g=-.75, \mathrm{BF}_{10}=28.16$ ).

Conclusions: The specific symptom of worry - not the diagnosis of an anxiety disorder - was associated with the most robust reductions in HRV, indicating that HRV may provide a transdiagnostic biomarker of worry. These results enhance understanding of the relationship between the cardiac autonomic nervous system and anxiety psychopathology, providing support for dimensional-trait models consistent with the Research Domain Criteria framework.
\end{abstract}

Keywords: Psychophysiology, Autonomic nervous system, ANS, Heart rate variability, HRV, Anxiety, Worry, Dimensional-trait models

\section{Background}

Anxiety disorders are the most prevalent of the psychiatric disorders [1], and the most costly [2]. Anxiety disorders carry a three to four-fold increased risk of cardiovascular disease (CVD) after accounting for gender, substance use, and depression, [3-5] and a two-fold increased risk for cardiac mortality [6-8]. Reductions in resting-state heart rate variability (HRV) reflect cardiac autonomic dysfunction, which plays a key role in the development of cardiovascular diseases. Although reductions in HRV may

\footnotetext{
* Correspondence: daniel.quintana@medisin.uio.no

'School of Psychology, University of Sydney, Sydney, Australia

${ }^{5}$ NORMENT, KG Jebsen Centre for Psychosis Research, Division of Mental Health and Addiction, University of Oslo, and Oslo University Hospital, Oslo, Norway

Full list of author information is available at the end of the article
}

provide a link between anxiety and ill health [9-13], past studies on anxiety disorders have reported contradictory findings. Here we sought to determine whether anxiety disorders or their symptoms spanning a nonclinical to clinical spectrum are associated with stronger relations with HRV.

HRV indexes the complex modification of heart rate over time and has become a widely used measure of autonomic control of heart rate. Low HRV is associated with a wide variety of psychological states, behaviours and conditions including reduced capacity for self-regulation, enhanced withdrawal behaviours, psychiatric illness and cardiovascular disease [14-19] leading us to suggest previously that HRV may help to elucidate the pathways linking mental and physical health [13]. Anxiety 
disorders have been characterised by low HRV [20] and two complementary models - polyvagal theory and neurovisceral integration - provide a platform on which these findings may be interpreted.

Polyvagal theory [21] links high resting-state HRV to social engagement and effective emotion regulation strategies, while low HRV is linked to withdrawal behaviours [21], a characteristic that may underpin many of the anxiety disorders. An alternative biobehavioural theory, the neurovisceral integration model $[22,23]$, further underscores the important inhibitory role of vagal activity in emotion regulation. This model outlines specific central and peripheral pathways that connect autonomic, attentional, and affective systems involved in emotion regulation. The model suggests that the integrity of these pathways may be compromised in anxiety disorders, such that the central and autonomic nervous systems are rigidly coupled, resulting in difficulty with disengaging from and inhibiting threat detection (e.g., hyper-vigilance, apprehension, avoidance, panic sensations, increases in heart rate, and decreases in HRV).

While studies on HRV in the anxiety disorders have reported contradictory findings, recent meta-analytic work has established that anxiety disorders are associated with poor autonomic function [24-26]. However, it remains unclear whether specific symptoms characteristic to the anxiety disorders or the disorder itself are characterised by the most robust associations. The finding that reduced HRV is a common feature of anxiety disorders (except perhaps obsessive-compulsive disorder; OCD) [20] may be interpreted within a dimensional-trait model of psychopathology [27] in which HRV reductions may reflect a failure to inhibit stereotypical fight-flight-freeze behavioural responses [21-23]. However, prior studies have typically focused on distinct nosological disorders rather than on transdiagnostic features of anxiety symptomatology. This is an important distinction because most psychological disorders are heterogeneous and symptoms may also be present in individuals that do not meet formal diagnostic criteria. Moreover, anxiety symptomatology is present in a wide range of psychiatric disorders. Therefore, in addition to comparing HRV in those with and without an anxiety disorder, we also determined whether participants high versus low on specific symptoms are associated with more robust reductions in HRV, regardless of diagnosis.

\section{Methods}

The current study was undertaken and reported in accordance with the Guidelines for Reporting on Articles on Psychiatry and Heart rate variability (GRAPH) [28], which provides a standardized set of criteria for reporting HRV studies in the biobehavioral sciences [see Additional file 1].

\section{Participants}

Ninety-one participants (mean age $=19.70$, age range: 17-29) were recruited for the present study including 27 who met diagnostic criteria for a DSM-IV anxiety disorder, and 64 control participants that did not meet any DSM-IV diagnostic criteria. Participants were recruited from an undergraduate participant pool and received course credit for participation. In a typical sample of undergraduate students, the prevalence of anxiety with clinical severity is relatively low. Therefore, in an effort to recruit participants experiencing high levels of anxiety, participants were recruited based on responses to the Depression Anxiety Stress Scales - Short Form (DASS-21) [29], a screening measure. At the beginning of semester, a cohort of undergraduate psychology students completed a battery of measures including the DASS-21, allowing for targeted recruitment. In the present study, targeted participants included those who scored in the severe-toextremely severe range on anxiety scale of the DASS-21. After providing written informed consent, all participants were administered the Anxiety Disorders Interview Schedule-IV for DSM-IV (ADIS-IV) [30] by one of two trained doctoral students (JAC or DSQ) to assess whether participants met DSM-IV criteria for an anxiety disorder. At the time of data collection, JAC had had extensive experience in administering the ADIS-IV through his studies as part of the doctorate of clinical psychology programme. DSQ was a $\mathrm{PhD}$ candidate in psychology and was provided with training and supervision in the administration of the ADIS-IV by JAC and MJAA, a clinical psychologist and senior lecturer in clinical psychology. All participants were provided with details of multiple mental health services they could access after testing, if required (e.g., if they reported significant distress). Control group participants did not meet criteria for any psychiatric disorder. All aspects of the study were approved by The University of Sydney's Human Research Ethics Committee.

Exclusion criteria for the study included self-reported chronic physical illness (e.g., cardiac illness, cancer, epilepsy, and diabetes mellitus), psychotropic medication, pregnancy or lactation, psychosis spectrum disorder, traumatic brain injury, substance or alcohol dependence. Participants were instructed not to consume caffeine or nicotine on the day of their laboratory visit. Body mass index (BMI; assessed with a standard scale and tape measure) and an assessment of alcohol intake using the Alcohol Use Disorders Identification Test (AUDIT) [31] were calculated due to previously reported relationships with HRV [19, 32].

\section{Measures}

Anxiety Disorders Interview Schedule for DSM-IV (ADIS-IV) [30]. The ADIS-IV is a semi-structured clinical interview based on DSM-IV-TR criteria, and is designed as a diagnostic tool for Axis-I disorders including anxiety 
and mood disorders. A clinical diagnosis was indicated by a clinical severity rating (CSR) of at least four on the clinical scale. The CSR is a rating made by the interviewing clinician (JAC or DSQ) on a 0 to 8 scale based on current symptom severity, distress, and interference $(0=$ none, $2=$ subclinical, $4=$ clinically significant, $6=$ moderately severe, $8=$ most severe).

Penn State Worry Questionnaire (PSWQ) [33]. The PSWQ is a 16-item self-report questionnaire designed to assess intensity and excessive worry. Items include "my worries overwhelm me" and "I worry all the time", and are presented on a 5-point Likert-type scale. The PSWQ has been shown to have good internal consistency [34] and well-established validity [35]. The internal consistency in this study was found to be good $(\alpha=.80)$.

Depression Anxiety Stress Scales - Short Form (DASS-21) [29]. The DASS-21 is a self report scale assessing levels of depression, anxiety, and stress over the previous week, and consists of three scales: depression (DASS-D), anxiety (DASS-A), and stress (DASS-S). Items are rated on a 4point Likert-type scale, with higher scores reflecting higher levels of depression, anxiety, or stress. The measure displays good-to-excellent reliability and validity [36]. Internal consistency of the three subscales was found to be good in the current study ( $\alpha$ 's ranged from .88-.95).

The State-Trait Anxiety Inventory (revised STAI-Y) [37]. The STAI-state subscale (STAI-S) is a brief scale, consisting of 20 items coded on a 4-point Likert scale, designed to measure transient emotional reactions. The STAI-S good concurrent validity $(\alpha=.75-.85)$ and testretest reliability in general: $\alpha=.73-.86$ [37].

\section{Definition of groups}

First, participants were grouped by diagnostic category according to whether diagnostic criteria were met for a primary diagnosis of an anxiety disorder as assessed by the ADIS-IV. While the analyses compared clinically anxious participants with non-anxious controls, associations with different dimensional indices of symptom severity, including depression, a symptom that is frequently comorbid with anxiety, were of primary interest [38]. Therefore, all participants, regardless of disorder diagnosis, were also divided into groups of high and low levels of symptom severity for worry, depression, anxiety, and stress, as defined by the PSWQ and the depression and anxiety subscales of the DASS-21 respectively. The cut-off for the high levels of worry symptoms from the PSWQ was 45 [39]. The anxiety and depression subscales of the DASS-21 were categorised into high symptom severity (severe to extremely severe; scores $\geq 21$ on the depression subscale, scores $\geq 15$ on the anxiety subscale, and scores $\geq 26$ on the stress subscale) and low symptom severity (normal to moderate; scores $\leq 20$ on the depression subscale, scores $\leq 14$ on the anxiety subscale, and scores $\leq 25$ on the stress subscale), using severity labels previously described based on normative data [29].

\section{Procedure}

After informed consent was obtained, participants were asked to complete the state-trait anxiety inventory for a measure of state anxiety (STAI-S). Thereafter, a brief medical and psychological history was obtained by trained doctoral students. Arterial blood pressure was also recorded. Participants then underwent the structured diagnostic interview (ADIS-IV), which varied in duration between 45 minutes and 90 minutes depending on clinical severity of the participant, after which a battery of questionnaires was completed. Electrocardiogram (ECG) electrodes were then attached and data was recorded for six minutes while participants were relaxed and in a seated position. No instructions were given to alter breathing to avoid confounding associated with visceral-medullary feedback.

\section{Physiological data recording and processing}

$\mathrm{Ag} / \mathrm{AgCl}$ electrodes were attached in a modified Lead-II formation (right clavicle and left iliac crest) with a reference electrode on the left clavicle, and connected to an ECG, which sampled at $1000 \mathrm{~Hz}$ (PowerLab 8/30: ADInstruments, Sydney, AUS). ECG R-R series was obtained by the identification of the zero-points after a local maximum of the first derivative series via dedicated software (HRV Module, Labchart, ADI). All relevant segments were visually inspected and corrected for false or undetected R-waves, movement artifacts, and ectopic beats using piecewise cubic spline interpolation, with assessor blind to group status. Participants exhibiting significant deviation from sinus rhythm and electromyographic or movement errors (i.e., $>0.5 \%$ of total beats) were excluded from the study. A frequency domain measure approximating the activity of respiratory sinus arrhythmia (high frequency, . $15 \mathrm{~Hz}-.40 \mathrm{~Hz} ; \mathrm{HF}$ ) was calculated by Fast Fourier Transform using Welch's Periodogram (window width 256s, $50 \%$ overlap, resampled at $4 \mathrm{~Hz}$ ). This measure of HRV was chosen as it best reflects parasympathetic modulation of the heart [40]. HRV metrics were calculated using Kubios (v2.0, Biosignal Analysis and Medical Imaging Group, University of Kuopio, Finland). HF-HRV violated Shapiro-Wilk's test for normality (all $p$ s $<.05$ ), so raw scores were log transformed.

\section{Statistical analysis}

Analyses were conducted using the "perfect $t$-test" script [41] and "stats" package in the R statistical environment (version 3.2.2). Welch's $t$-tests and Pearson's Chi-square test compared differences between groups on relevant demographic variables, including age and gender, to 
assess whether groups differed on common factors known to impact HRV. Welch's $t$-tests were also used to compare HF HRV between groups. The common language effect size was computed, giving the probability that one random group observation is higher than another random observation from the other group [42]. Hedges' $g$ was also calculated as an effect size measure; this measure is better suited to studies with small sample sizes than the Cohen's $d$ measure [43]. Bayes Factors $\left(\mathrm{BF}_{10}\right)$ were calculated to quantify evidence for the alternative hypothesis (H1) relative to the null hypothesis (H0) [44] with a noninformative Jeffreys prior placed on the variance of the normal population and a Cauchy prior placed on the standardized effect size. The Bayes factor $r$-scale prior (not to be confused with Pearson's $r$ correlation coefficient) was set at 0.5 , as a small effect size was anticipated. $\mathrm{A} \mathrm{BF}_{10}$ value $<0.33$ provides strong or 'substantial' evidence for the null hypothesis, over 3 provides strong evidence for the alternative hypothesis and between 0.33 and 3 provides only anecdotal support either way [45]. To investigate the relationship between HRV and variables of interest, correlational analyses were conducted across all participants with two-tailed Pearson correlations and Bayes Factors (putting a uniform prior on rho) using the JASP statistical package (version 0.7.5.5) [46]. The importance of reporting effect sizes regardless of statistical significance has been highlighted previously $[5,47,48]$, and these recommendations are followed here. The correlation coefficient was interpreted as large when $r=.5$, medium when $r=.3$, and small when $r=.10$, while Hedges' $g$ was interpreted as large when $g=.80$, medium when $g=.50$, and small when $g=.20$.

\section{Results}

\section{Participant characteristics}

After blinded inspection of the ECG data, eight participants were excluded due to artefacts or significant deviations from sinus rhythm that comprised more than $0.5 \%$ of total beats over the six-minute recording (ECG data available upon request), leaving a sample of 83 participants. Table 1 presents the participant characteristics for the control and clinical groups. There were no significant differences between groups on age, gender, and alcohol use. The clinical group had a significantly lower BMI than the control group ( $p=0.04$, Hedges' $g=0.44$; Table 1), however, a $\mathrm{BF}_{10}$ of 1.78 indicates the data are only 1.78 times more likely under the alternative hypothesis (than under the null hypothesis) providing only anecdotal evidence for the alternative hypothesis. Moreover, BMI was not significantly correlated with HRV $(r=0.02$; $95 \%$ CI: -.2 to $.24 ; p=.84$ ), with the $\mathrm{BF}$ providing substantial evidence that these variables are not related $\left(\mathrm{BF}_{10}=.14\right)$. These findings suggest that group differences in BMI are unlikely to contribute to differences in HRV in the present
Table 1 Participant demographic and symptom characteristics

\begin{tabular}{llll}
\hline & Clinical $(n=25)$ & Control $(n=58)$ & $p$-values \\
\hline Age in years $^{\mathrm{a}}$ & $19.71(2.8)$ & $19.56(2.62)$ & .83 \\
N of females (\%) & 76 & 58.6 & .13 \\
AUDIT & $6.44(5.87)$ & $7.84(5.92)$ & .32 \\
Systolic BP & $119.32(14.53)$ & $122.19(13.42)$ & .4 \\
Diastolic BP $^{b}$ & $79.76(13.15)$ & $75.81(11.18)$ & .2 \\
BMI $^{\text {b }}$ & $21.31(2.97)$ & $23.01(4.16)$ & .04 \\
Symptom Measures & & & \\
PSWQ $^{c}$ & $60.5(11.77)$ & $42(11.85)$ & $<.001$ \\
DASS D $^{d}$ & $20.5(12.75)$ & $6.93(8.41)$ & $<.001$ \\
DASS A $^{d}$ & $18.67(10.74)$ & $4.93(6.23)$ & $<.001$ \\
DASS S $^{d}$ & $24.67(10)$ & $10.62(8.16)$ & $<.001$ \\
STAI S $^{\mathrm{e}}$ & $47.12(11.37)$ & $32.96(7.74)$ & $<.001$ \\
\hline
\end{tabular}

Note: Means and standard deviations (in parentheses) are presented for continuous data; AUDIT Alcohol Use Disorders Identification Test, BMI Body mass index, PSQW Penn State Worry Questionnaire, DASS D Depression, Anxiety and Stress Scale (short-form) depression subscale, DASS21-A Depression, Anxiety and Stress Scale (short-form) anxiety subscale, DASS A Depression, Anxiety and Stress Scale (short-form) stress subscale, STAI S State-Trait Anxiety Inventory State scale. ${ }^{\mathrm{a}}$ Clinical $n=24$, Control $n=55$; ${ }^{\mathrm{b}}$ Clinical $n=25$, Control $\mathrm{n}=57$; ${ }^{\mathrm{C} C l i n i c a l}$ $n=24$, Control $n=57$; ${ }^{\mathrm{d}}$ Clinical $n=24$, Control $n=58$; ${ }^{\mathrm{e}}$ Clinical $n=25$, Control $n=55$

sample. As expected, clinical participants exhibited higher scores on all psychological measures of anxiety, depression, worry, and stress, relative to controls (all $p$ 's $<.001$, see Table 1).

Overall, 25 participants met diagnostic criteria for a primary anxiety disorder, including PD $(n=3), \operatorname{GAD}(n=8)$, $\operatorname{PTSD}(n=1)$, Social anxiety disorder $(n=12)$, and Obsessive compulsive disorder $(n=1)$. The mean CSR of these 25 participants was $4.92(\mathrm{SD}=1.08)$ indicating clinical severity in this sample to be mild-to-moderate. While 17 of 25 clinical participants did not suffer from a comorbid anxiety disorder, six participants suffered from two anxiety disorders, and two participants suffered from three anxiety disorders. Division of groups were defined by symptom severity as follows: high depression ( $n=17$; including $n=12$ from the clinical group) and low depression $(n=66$; including $n=13$ from the clinical group); high anxiety ( $n=$ 20; including $n=16$ from the clinical group), low anxiety ( $n=63$; including $n=9$ from the clinical group), and high stress ( $n=15$; including $n=12$ from the clinical group) and low stress $(n=67$; including $n=12$ from the clinical group); and high worry ( $n=41$; including 22 from the clinical group) and low worry ( $n=42$; including $n=3$ from the clinical group). These divisions were based on well-established cut-offs described earlier. Given the dimensional nature of anxiety and depression [38], and that some individuals report high levels of depression/anxiety without meeting diagnostic criteria, these groups were defined regardless of clinical status. Of note, some participants both met criteria for a primary anxiety disorder, and 
also fell in the low anxiety group, which may seem counterintuitive. These participants likely reflect those who met criteria for an anxiety disorder without symptoms of somatic anxiety as assessed by the anxiety subscale (e.g., GAD).

\section{Differences in HRV between groups}

The clinical anxiety group displayed lower HF-HRV $(M=6.34, S D=.99, n=25)$ than controls $(M=6.71$, $S D=.97, n=58)$ at trend levels $[t(44.89)=-1.57 ; p=.1$; Hedges' $g=-.37$; 95 \% CI $(-0.85,0.1)$; Fig. 1a]. According to the common language effect size, the likelihood that the HF HRV of a random person in the clinical anxiety group is smaller than the HF HRV of a random person in the control group is $60 \%$. The $\mathrm{BF}_{10}$ was 0.84 indicating that the data are 0.84 times more likely under the alternative hypothesis, than under the null hypothesis, providing anecdotal evidence for the null hypothesis.

The high worry group displayed significantly reduced HF-HRV $(M=6.24, S D=.9, n=41)$ relative to the low worry group $(M=6.94, S D=.96, n=40 ; t(78.33)=-3.4$, $p=.001$; Fig. 1b), a finding associated with a medium effect size [Hedges' $g=-.75,95 \%$ CI $(-1.2,-0.3)]$. According to the common language effect size, the likelihood that the HF HRV of a random person in the worry group is smaller than the HF HRV of a random person in the low worry group is $70 \%$. The $\mathrm{BF}_{10}$ of 28.16 further indicates that the data are 28.16 times more likely under the alternative hypothesis, than under the null hypothesis, providing strong evidence for alternative hypothesis.

The high anxiety group displayed reduced HF-HRV $(M=6.23, S D=0.93, n=20)$ relative to the low anxiety group ( $M=6.7, S D=0.98, n=62)$, a finding that bordered the threshold for significance $[t(33.32)=-1.94, p=.06$, Hedges' $g=-.48$; 95 \% CI (-1.01, 0.01); Fig. 1c]. According to the common language effect size, the likelihood that the HF HRV of a random person in the high anxiety group is smaller than the HF HRV of a random person in the low anxiety group is $64 \%$. The $\mathrm{BF}_{10}$ was 1.42 indicating that the data are 1.42 times more likely under the alternative hypothesis, than under the null hypothesis, providing only anecdotal evidence for the alternative hypothesis.

There was no significant difference in HF-HRV between those categorised with high $(M=6.43, S D=0.92, n=17)$ and low $(M=6.63, S D=1, n=65)$ levels of depression severity $[\mathrm{t}(26.73)=-0.75, p=0.46$ Hedges' $g=-.19 ; 95 \% \mathrm{CI}$ (-.74, 0.33); Fig. 1d]. According to the common language effect size, the likelihood that HF-HRV of a person selected at random from the high depression group is smaller than one selected at random from the low depression group is $56 \%$. The $\mathrm{BF}_{10}$ was 0.44 , indicating that the data are only 0.44 times more likely under the alternative hypothesis, than under the null hypothesis, providing only anecdotal evidence for the null hypothesis.

There was also no difference in HF-HRV between those categorised as high $(M=6.35, S D=0.79, n=15)$ and low $(M=6.64, S D=1.02, n=67)$ on stress $[t(25.62)=-1.2$, $p=0.240$, Hedges' $g=-0.29,95 \%$ CI $(-0.91,0.22)$; Fig. 1e]. According to the common language effect size, the likelihood that the HF-HRV of a random person in the high stress group is smaller than the HF HRV of a random person in the low stress group is $59 \%$. The $\mathrm{BF}_{10}$ was 0.62 , which indicates the data are 0.62 times more likely under the alternative hypothesis, than under the null hypothesis, providing only anecdotal evidence for the null hypothesis.

\section{Associations between symptom severity measures and HRV}

Bivariate correlations examined the relationship between symptom severity measures and HF-HRV at rest. The

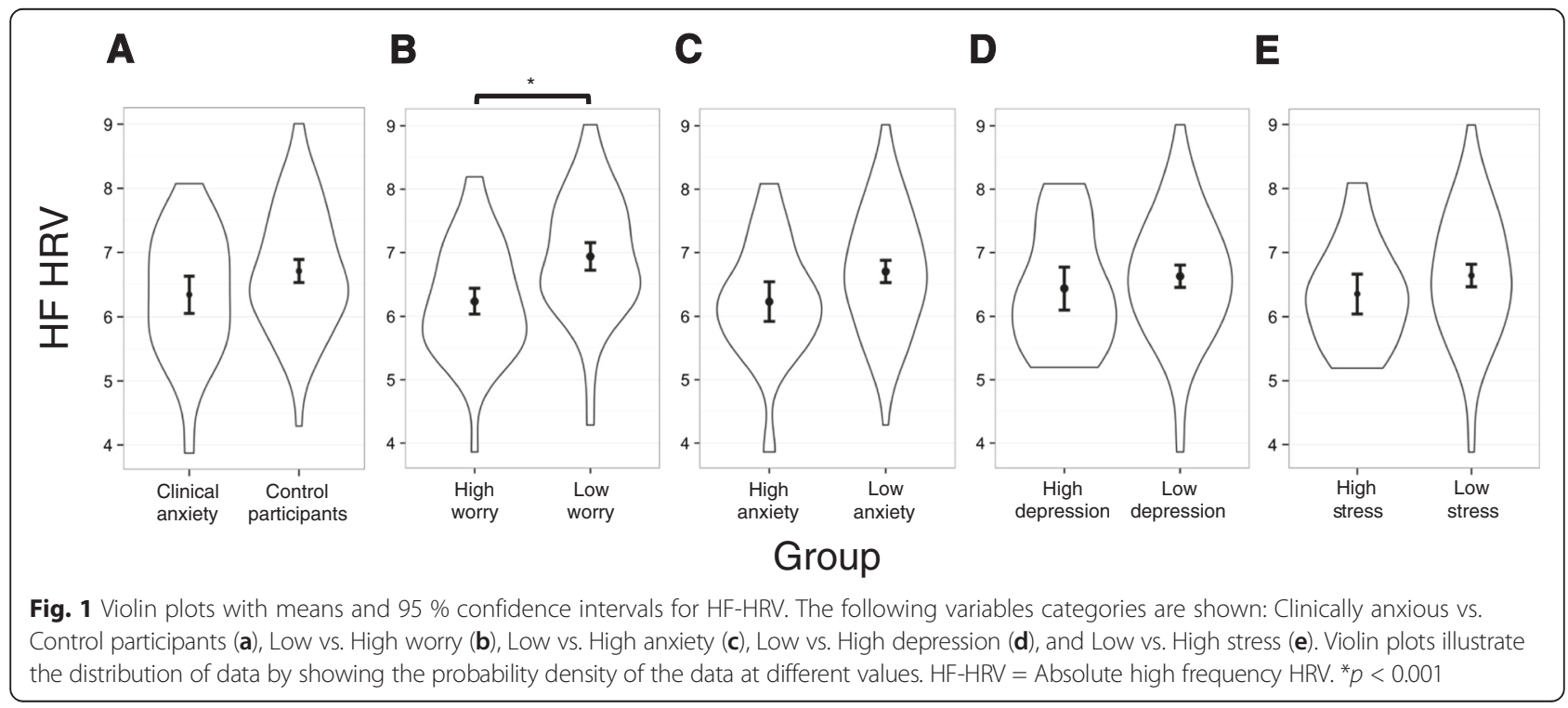


relationship between HF-HRV and worry (indexed by the PSWQ) was significantly inversely correlated $(r=-0.31$; 95 \% CI: -.49 to -.10; $p=.01$; Fig. 2a), with the BF providing substantial evidence that these variables are inversely associated $\left(\mathrm{BF}_{10}=5.9\right)$. Pearson's correlation coefficient was indicative of a moderate effect size. There was no significant relationship between HF-HRV and anxiety $(r=-0.21 ; 95 \%$ CI: -.41 to $-.01 ; p=.06$; Fig. $2 \mathrm{~b})$ or stress $(r=-0.19 ; 95 \%$ CI: -.39 to $.03 ; p=.09$; Fig. 2 c), with the BFs providing only anecdotal evidence that these variables are negatively associated (anxiety $\mathrm{BF}_{10}=.83$; stress $\mathrm{BF}_{10}=.58$ ). There was also no significant relationship between HF-HRV and depression $(r=-0.14 ; 95 \%$ CI: -.34 to $.08 ; p=.23$; Fig. $2 \mathrm{~d}$ ), and the BF provided substantial evidence that these variables are not related $\left(\mathrm{BF}_{10}=.28\right)$.

\section{Discussion}

This study examined whether HRV in participants who fulfilled DSM-IV criteria for an anxiety disorder differed from control participants at resting state, and whether HRV reductions were more reliably associated with measures of depression, anxiety, stress, and worry, consistent with dimensional-trait models of psychopathology. HRV was reduced in clinically anxious participants relative to controls, although this difference only bordered on statistical significance. While prior research has demonstrated that HRV is reduced across anxiety disorders, there are variations across disorders in the degree to which this effect has been observed [25]. In fact, a recent study [26] across common mental disorders has demonstrated that only generalised anxiety disorder may display HRV reductions after many potential confounding factors are controlled. Heterogeneity across multiple anxiety disorders is one explanation for borderline findings between those with and without an anxiety disorder.

In the present study, all measures of symptom severity had an inverse relationship with HRV. However, only the
PSWQ, an index of worry and cardinal feature of GAD, was observed to correlate significantly with HRV. These correlational analyses were complimented by betweengroup findings indicating that high worriers displayed significantly reduced HRV relative to low worriers, a finding associated with a large effect size. These findings were also complimented by strong and substantial evidence from Bayesian analyses, and provide convergent support for results from past studies, which have indicated that generalised anxiety disorder may be characterised by the most robust reductions in HRV $[26,49]$. Our recent metaanalysis on the association between the anxiety disorders and HRV [25] also observed that HRV reductions in GAD were associated with a large effect size, while others have observed a significant inverse relationship between anxiety symptom severity in GAD patients and HRV [50]. Interestingly, recent evidence has also linked functional brain mechanisms associated with worry and rumination in GAD patients to reductions in HRV [51]. The source of worry in high worriers is not an external stressors, but cognitions about future threats [52]. Accordingly, pathological worry is distinguished by its chronicity, in contrast with more phasic forms of anxiety, such as panic [53]. Moreover, it has been suggested that anxiety in GAD is recognised as reflecting a long-term trait, or anxious temperament $[54,55]$. It may be the chronic nature of worry symptomatology that leads to long-term withdrawal of the parasympathetic nervous system and persistent HRV reductions $[26,56]$. The current finding of reduced HRV in high worriers is important considering the literature documenting the role of worry and GAD in cardiovascular risk [57].

\section{Study limitations}

Some limitations of the present research should be noted. First, the specific impact of nosologically distinct anxiety disorders on HRV was not assessed. However, recent research on anxiety and HRV has also suggested that diminished HRV may represent a shared feature of
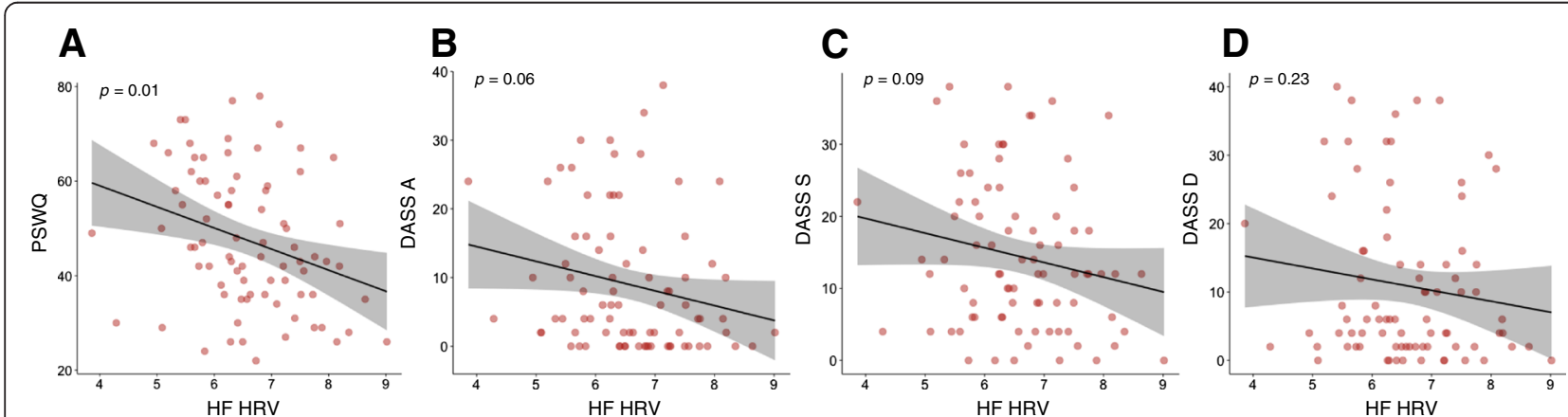

Fig. 2 A scatterplot visualising the association between HRV and worry (a), anxiety (b), stress (c), and depression (d) symptom severity. A line of best fit with $95 \%$ confidence region was overlaid on the scatterplots to illustrate data trends. DASS A = DASS anxiety score; DASS S = DASS stress score, DASS D = DASS depression score; PSWQ = Penn State worry questionnaire score 
anxiety disorders [50]. HRV may therefore provide a transdiagnostic psychophysiological marker of anxiety psychopathology. The investigation of common autonomic features across anxiety disorders - such as HRV - may also be a more ecologically valid method given the high level of co-morbidity across distinct disorders [58].

Second, while it is known that respiration parameters including rate and depth can affect $\operatorname{HRV}[59,60]$, these factors were not accounted for throughout the experiment. While a commonly employed resolution to this problem has been to use paced breathing (e.g., [61]), the control of breathing may itself change HRV due to cortical involvement and adjusting visceral-medullary feedback [62]. Furthermore, some studies have reported that paced breathing may not provide any additional insights into autonomic function, over that provided when participants are spontaneously breathing [63-65].

\section{Conclusions}

The present research indicates that although resting state HRV is reduced in individuals diagnosed with an anxiety disorder, the dimensional symptom of worry may actually be driving the observed HRV reductions, at least in the anxiety disorders. This finding provides support for several lines of research leading to proposals characterising vagal function - indexed by HF-HRV - as a physical pathway linking mental and physical health $[13,66]$. Vagal function is considered to reflect a physical link because it not only appears to lay a physiological foundation from which psychological flexibility may arise, but it also has been shown to play an important regulatory role over a variety of physiological systems, including the sympathetic nervous system, the hypothalamic-pituitary-adrenal axis and inflammatory processes. Furthermore, our findings lend support to a recent suggestion [67] that HRV may be considered to index certain dimensional-traits underpinning psychiatric disorders that transcend diagnostic labels. In this regard, findings from the present study demonstrate that HRV indexes worry in individuals spanning the nonclinical to clinical spectrum. Future research should explore the impact of worry symptoms on HRV in other psychopathologies, such as major depressive disorder. The establishment of HRV as reliable transdiagnostic biomarker for worry may help facilitate the development of novel treatments (e.g., Non-invasive transcutaneous vagus nerve stimulation) and the identification of specific subgroups that are more likely to respond to such treatments. Finally, our results provide support for alternative frameworks for understanding psychiatric disorders, such as the Research Domain Criteria (or RDoC), and identify HRV as a particularly useful index of psychopathology that may index cognitive dysfunctions (i.e. excessive worry) leading to subsequent 'wear and tear' on the human body.

\section{Additional file}

Additional file 1: Guidelines for Reporting on Articles on Psychiatry and Heart rate variability (GRAPH) checklist. A checklist of recommended items to report in a biobehavioral heart rate variability study. (PDF 64 kb)

\section{Abbreviations}

ADIS-IV, Anxiety Disorders Interview Schedule for DSM-IV; BF, Bayes factor; CSR, Clinical severity rating; CVD, Cardiovascular disease; OCD, Obsessivecompulsive disorder; DASS-21, Depression Anxiety Stress Scales - Short form; DASS-A, Depression Anxiety Stress Scales - Anxiety scale; DASS-D, Depression Anxiety Stress Scales - Depression scale; DASS-S, Depression Anxiety Stress Scales - Stress scale; GAD, Generalized anxiety disorder; HF, High frequency; HRV, Heart rate variability; PD, Panic disorder; PSWQ, Penn State Worry Questionnaire; RDoC, Research domain criteria; GRAPH, Guidelines for Reporting on Articles on Psychiatry and Heart rate variability; BMI, body mass index; AUDIT Alcohol Use Disorders Identification Test; STAI-S, State-Trait Anxiety Inventory; ECG, electrocardiogram.

Availability of data and materials

The data will not be made publically available in order to protect participant identity but is available upon request to Dr. Daniel Quintana (daniel.quintana@medisin.uio.no).

\section{Authors' contributions}

DQ, MA, and AK designed the study. DQ and JC acquired the data. DQ, JC and $\mathrm{JH}$ analysed and interpreted the data. $\mathrm{JC}$ drafted the first version of the manuscript and all other authors revised it for important intellectual content, gave final approval of the version to be published, and agree to be accountable for all aspects of the work.

\section{Competing interests}

The authors have no financial or non-financial competing interests to declare.

\section{Consent for publication}

Not applicable.

\section{Ethics approval and consent to participate}

The research was performed in accordance with the Declaration of Helsinki and was approved by the University of Sydney Human Research Ethics committee.

\section{Author details}

${ }^{1}$ School of Psychology, University of Sydney, Sydney, Australia. ${ }^{2}$ Division of Cardiology, Poznań University of Medical Sciences, Poznań, Poland.

${ }^{3}$ Discipline of Psychiatry, University of Sydney, Sydney, Australia. ${ }^{4}$ Department of Psychology, Swansea University, Swansea, UK. ${ }^{5}$ NORMENT, KG Jebsen Centre for Psychosis Research, Division of Mental Health and Addiction, University of Oslo, and Oslo University Hospital, Oslo, Norway. ${ }^{6}$ NORMENT, KG Jebsen Centre for Psychosis Research, Building 49, Oslo University Hospital, Ullevål, Kirkeveien 166, PO Box 4956, Nydalen N- 0424 Oslo, Norway.

Received: 4 December 2015 Accepted: 26 May 2016

Published online: 03 June 2016

\section{References}

1. Kessler RC, Angermeyer M, Anthony JC, Deg R, Demyttenaere K, Gasquet I, G Deg, Gluzman S, Gureje O, Haro JM, et al. Lifetime prevalence and age-ofonset distributions of mental disorders in the World Health Organization's World Mental Health Survey Initiative. World Psychiatry. 2007;6(3):168-76.

2. Kessler RC, Greenberg PE. The economic burden of anxiety and stress disorders. Neuropsychopharmacology: The fifth generation of progress. 2002;981-992.

3. Härter MC, Conway KP, Merikangas KR. Associations between anxiety disorders and physical illness. Eur Arch Psychiatry Clin Neurosci. 2003;253(6): 313-20. 
4. Vogelzangs N, Seldenrijk A, Beekman AT, van Hout HP, de Jonge P, Penninx BW. Cardiovascular disease in persons with depressive and anxiety disorders. J Affect Disord. 2010;125(1-3):241-8.

5. Kemp AH, Brunoni AR, Nunes MA, Santos IS, Goulart AC, Ribeiro AL, Benseñor IM, Lotufo PA: The association between mood and anxiety disorders, and coronary heart disease in Brazil: a cross-sectional analysis on the Brazilian longitudinal study of adult health (ELSA-Brasil). Frontiers in psychology 2015;6:187.

6. Roest AM, Martens EJ, de Jonge P, Denollet J. Anxiety and risk of incident coronary heart disease: a meta-analysis. J Am Coll Cardiol. 2010; 56(1):38-46.

7. Janszky I, Ahnve S, Lundberg I, Hemmingsson T. Early-onset depression, anxiety, and risk of subsequent coronary heart disease: 37-year follow-up of 49,321 young Swedish men. J Am Coll Cardiol. 2010;56(1):31-7.

8. Shibeshi WA, Young-Xu Y, Blatt CM. Anxiety worsens prognosis in patients with coronary artery disease. J Am Coll Cardiol. 2007;49(20):2021-7.

9. Malpas SC. Sympathetic nervous system overactivity and its role in the development of cardiovascular disease. Physiol Rev. 2010;90(2):513-57.

10. Triposkiadis F, Karayannis G, Giamouzis G, Skoularigis J, Louridas G, Butler J. The sympathetic nervous system in heart failure: physiology, pathophysiology, and clinical implications. J Am Coll Cardiol. 2009;54(19):1747-62.

11. Esler M, Kaye D. Sympathetic nervous system activation in essential hypertension, cardiac failure and psychosomatic heart disease. J Cardiovasc Pharmacol. 2000;35:S1-7.

12. Dekker JM, Crow RS, Folsom AR, Hannan PJ, Liao D, Swenne CA, Schouten EG. Low heart rate variability in a 2-minute rhythm strip predicts risk of coronary heart disease and mortality from several causes - The ARIC study. Circulation. 2000;102(11):1239-44.

13. Kemp AH, Quintana DS. The relationship between mental and physical health: Insights from the study of heart rate variability. Int J Psychophysiol. 2013;89(3):288-96

14. Thayer JF, Yamamoto SS, Brosschot JF. The relationship of autonomic imbalance, heart rate variability and cardiovascular disease risk factors. Int J Cardiol. 2010; 141(2):122-31.

15. Kemp AH, Quintana DS, Gray MA, Felmingham KL, Brown K, Gatt JM. Impact of depression and antidepressant treatment on heart rate variability: a review and meta-analysis. Biol Psychiatry. 2010;67(11):1067-74.

16. Geisler F, Kubiak T, Siewert K, Weber H. Cardiac vagal tone is associated with social engagement and self-regulation. Biol Psychol. 2013;93(2):279-86.

17. Quintana DS, Westlye LT, Kaufmann T, Rustan $\varnothing$, Brandt CL, Haatveit B, Steen NE, Andreassen OA: Reduced heart rate variability in schizophrenia and bipolar disorder compared to healthy controls. Acta Psychiatrica Scandinavica 2016;133(1):44-52

18. Alvares GA, Quintana DS, Hickie IB, Guastella AJ: Autonomic nervous system dysfunction in psychiatric disorders and the impact of psychotropic medications: a systematic review and meta-analysis. J Psychiatry Neurosci 2016 41(2):89-104

19. Quintana DS, McGregor IS, Guastella AJ, Malhi GS, Kemp AH. A meta-analysis on the impact of alcohol dependence on short-term resting-state heart rate variability: implications for cardiovascular risk. Alcohol Clin Exp Res. 2013; 37(Suppl 1(s1)):E23-29.

20. Chalmers JA, Quintana DS, Maree J, Abbott A, Kemp AH: Anxiety disorders are associated with reduced heart rate variability: a meta-analysis. Frontiers in psychiatry 2014, 5

21. Porges SW. The polyvagal theory: Neurophysiological foundations of emotions, attachment, communication, and self-regulation. New York, NY: W. W. Norton \& Company; 2011.

22. Thayer JF, Lane RD. A model of neurovisceral integration in emotion regulation and dysregulation. J Affect Disord. 2000;61(3):201-16.

23. Thayer JF, Lane RD. Claude Bernard and the heart-brain connection: Further elaboration of a model of neurovisceral integration. Neurosci Biobehav Rev. 2009;33(2):81-8

24. Alvares GA, Quintana DS, Hickie IB, Guastella AJ: Autonomic Nervous System Dysfunction in Psychiatric Disorders and the Impact of Psychotropic Medications: A Systematic Review and Meta-Analysis. J Psychiatry Neurosci 2016; 41(2):89-104.

25. Chalmers J, Quintana DS, Abbott MJ, Kemp AH. Anxiety disorders are associated with reduced heart rate variability: A meta-analysis. Affective Disorders Psychosomatic Res. 2014;5:80.

26. Kemp AH, Brunoni AR, Santos IS, Nunes MA, Dantas EM, De Fiqueiredo RC, Pereira AC, Ribeiro AL, Mill JG, Andreão RV: Effects of depression, anxiety, comorbidity, and antidepressants on resting-state heart rate and its variability: an ELSA-Brasil cohort baseline study. American Journal of Psychiatry 2014; 171(12):1328-1334

27. Beauchaine TP, Thayer JF. Heart rate variability as a transdiagnostic biomarker of psychopathology. Int J Psychophysiol. 2015;98(2):338-50.

28. Quintana DS, Alvares GA, Heathers JA: Guidelines for Reporting Articles on Psychiatry and Heart rate variability (GRAPH): Recommendations to advance research communication. Transl Psychiatry 2016;6(e803):1-10.

29. Lovibond SH, Lovibond PF: Manual for the Depression Anxiety Stress Scales, 2nd. Ed. edn. Sydney: Psychology Foundation; 1995.

30. DiNardo PA, Brow TA, Barlow DH. Anxiety Disorders Interview Schedule for DSM-IV: Life Time Version: Client Interview Schedule. 1994. Graywind.

31. Saunders JB, Aasland OG, Babor TF, de la Fuente JR, Grant M. Development of the alcohol use disorders identification test (AUDIT): WHO collaborative project on early detection of persons with harmful alcohol consumptionll. Addiction. 1993;88(6):791-804.

32. Karason K, Mølgaard H, Wikstrand J, Sjöström L. Heart rate variability in obesity and the effect of weight loss. Am J Cardiol. 1999;83(8):1242-7.

33. Meyer TJ, Miller ML, Metzger RL, Borkovec TD. Development and validation of the Penn State worry questionnaire. Behav Res Ther. 1990; 28(6):487-95.

34. Molina S, Borkovec T. The Penn State Worry Questionnaire: Psychometric properties and associated characteristics. In: Davey GCL, Tallis F, editors. Worrying: Perspectives on theory, assessment and treatment. Oxford, England: John Wiley \& Sons; 1994. p. 265-83.

35. Brown TA, Antony MM, Barlow DH. Psychometric properties of the Penn State Worry Questionnaire in a clinical anxiety disorders sample. Behav Res Ther. 1992;30(1):33-7.

36. Antony MM, Bieling PJ, Cox BJ, Enns MW, Swinson RP. Psychometric properties of the 42-item and 21-item versions of the Depression Anxiety Stress Scales in clinical groups and a community sample. Psychol Assess. 1998;10(2):176.

37. Spielberger C, Gorsuch RL, Lushene R, Vagg P, Jacobs G. Manual for the StateTrait Anxiety Inventory. Palo Alto, CA: Consulting Psychologists Press; 1983.

38. Haslam N. Categorical versus dimensional models of mental disorder: The taxometric evidence. Aust N Z J Psychiatry. 2003;37(6):696-704.

39. Behar $E$, Alcaine $O$, Zuellig AR, Borkovec T. Screening for generalized anxiety disorder using the Penn State Worry Questionnaire: A receiver operating characteristic analysis. J Behav Ther Exp Psychiatry. 2003;34(1):25-43.

40. Camm A, Malik M, Bigger J, Breithardt G, Cerutti S, Cohen R, Coumel P, Fallen E, Kennedy $\mathrm{H}$, Kleiger $\mathrm{R}$. Heart rate variability: standards of measurement, physiological interpretation and clinical use. Task Force of the European Society of Cardiology and the North American Society of Pacing and Electrophysiology. Circulation. 1996;93(5):1043-65.

41. Lakens D: The perfect t-test (version 1.0.0). Retrieved from https://github. com/Lakens/perfect-t-test. doi:10.5281/zenodo.17603

42. McGraw KO, Wong S. A common language effect size statistic. Psychol Bull. 1992;111(2):361

43. Cumming G: Understanding the new statistics: Effect sizes, confidence intervals, and meta-analysis. New York, NY: Routledge; 2013.

44. Morey RD, Rouder JN. Bayes factor approaches for testing interval null hypotheses. Psychol Methods. 2011;16(4):406.

45. Dienes Z. Using Bayes to get the most out of non-significant results. Frontiers Psychology. 2014;5(781):1-17.

46. JASP Team. JASP (Version 0.7.5.5). 2016

47. Thompson B. Statistical significance tests, effect size reporting and the vain pursuit of pseudo-objectivity. Theory Psychology. 1999;9(2):191-6.

48. Rosnow RL, Rosenthal R. Statistical procedures and the justification of knowledge in psychological science. Am Psychol. 1989;44(10):1276.

49. Kemp AH, Quintana DS, Felmingham KL, Matthews S, Jelinek HF. Depression, comorbid anxiety disorders, and heart rate variability in physically healthy, unmedicated patients: implications for cardiovascular risk. PLoS One. 2012;7(2), e30777.

50. Pittig A, Arch JJ, Lam CW, Craske MG. Heart rate and heart rate variability in panic, social anxiety, obsessive-compulsive, and generalized anxiety disorders at baseline and in response to relaxation and hyperventilation. Int J Psychophysiol. 2013;87(1):19-27.

51. Makovac E, Meeten F, Watson DR, Herman A, Garfinkel SN, Critchley HD, Ottaviani C: Alterations in Amygdala-Prefrontal Functional Connectivity Account for Excessive Worry and Autonomic Dysregulation in Generalized Anxiety Disorder. Biol Psychiatry 2015. 
52. Barlow DH. Anxiety and its disorders: The nature and treatment of anxiety and panic. New York: NY: Guilford Press; 2004.

53. Weisberg RB: Overview of generalized anxiety disorder: epidemiology, presentation, and course. Journal of clinical psychiatry 2009:70(s2):4-9.

54. Akiskal $\mathrm{H}$. Toward a definition of generalized anxiety disorder as an anxious temperament type. Acta Psychiatr Scand. 1998;98(s393):66-73.

55. Rickels K, Schweizer E. Long-term treatment of anxiety disorders: Maintenance treatment studies in anxiety disorders: Some methodological notes. Psychopharmacol Bull. 1995;31:115-23.

56. Brosschot JF, Gerin W, Thayer JF. The perseverative cognition hypothesis: a review of worry, prolonged stress-related physiological activation, and health. J Psychosom Res. 2006;60(2):113-24.

57. Tully PJ, Cosh SM, Baune BT. A review of the affects of worry and generalized anxiety disorder upon cardiovascular health and coronary heart disease. Psychol Health Med. 2013:18(6):627-44.

58. Kessler RC, Chiu WT, Demler O, Merikangas KR, Walters EE. Prevalence, severity, and comorbidity of 12-month DSM-IV disorders in the National Comorbidity Survey Replication. Arch Gen Psychiatry. 2005;62(6):617-27.

59. Schipke J, Arnold G, Pelzer M. Effect of respiration rate on short-term heart rate variability. J Clinical and Basic Cardiology. 1999;2(1):92-5.

60. Pöyhönen M, Syväoja S, Hartikainen J, Ruokonen E, Takala J. The effect of carbon dioxide, respiratory rate and tidal volume on human heart rate variability. Acta Anaesthesiol Scand. 2004;48(1):93-101.

61. Sato N, Miyake S, Akatsu J, Kumashiro M. Power spectral analysis of heart rate variability in healthy young women during the normal menstrual cycle. Psychosom Med. 1995:57(4):331-5.

62. Tripathi K. Respiration and heart rate variability: A review with special reference to its application in aerospace medicine. Indian J Aerospace Med. 2004;48(1):64-75.

63. Patwardhan A, Evans J, Bruce E, Knapp C. Heart rate variability during sympatho-excitatory challenges: comparison between spontaneous and metronomic breathing. Integrative Physiological Behavioral Science. 2001; 36(2):109-20.

64. Patwardhan AR, Evans JM, Bruce EN, Eckberg DL, Knapp CF. Voluntary control of breathing does not alter vagal modulation of heart rate. J Appl Physiol. 1995;78(6):2087-94.

65. Quintana DS, Heathers JA. Considerations in the assessment of heart rate variability in biobehavioral research. Frontiers Psychology. 2014;5.

66. Verkuil B, Brosschot JF, Gebhardt WA, Thayer JF. When worries make you sick: a review of perseverative cognition, the default stress response and somatic health. J Experimental Psychopathology. 2010;1(1):87-118.

67. Beauchaine TP, Thayer JF: Heart rate variability as a transdiagnostic biomarker of psychopathology. Int J Psychophysiol 2015;98(2):338-350.

\section{Submit your next manuscript to BioMed Central and we will help you at every step:}

- We accept pre-submission inquiries

- Our selector tool helps you to find the most relevant journal

- We provide round the clock customer support

- Convenient online submission

- Thorough peer review

- Inclusion in PubMed and all major indexing services

- Maximum visibility for your research

Submit your manuscript at www.biomedcentral.com/submit

CBiomed Central 